\title{
Development of Direct Manipulation Interface for Collaborative VR/MR Workspace
}

\author{
Hiroshi Sasaki ${ }^{1}$, Tadayuki Takeda ${ }^{2}$, Masataka Imura $^{2}$, Yoshihiro Yasumuro ${ }^{2}$, \\ Yoshitsugu Manabe ${ }^{2}$, and Kunihiro Chihara ${ }^{2}$ \\ ${ }^{1}$ Information Science and Technology Center, Kobe University, \\ 1-1, Rokkodai, Nada, Kobe, Hyogo, 657-8501, Japan \\ sasakiakobe-u.ac.jp \\ http: / /www.istc.kobe-u.ac.jp/ \\ ${ }^{2}$ Graduate School of Information Science, Nara Institute of Science and Technology, \\ 8916-5, Takayama, Ikoma, Nara, 630-0192, Japan \\ \{tadayu-t, imura, yasumuro, manabe, chihara\}@is.naist.jp \\ http://chihara.naist.jp/index.html
}

\begin{abstract}
Our research projects aim to connect various VR/MR platforms and to realize the seamless collaborative works with the intuitive operations whenever and wherever users like. In order to realize an ideal collaborative workspace, an effective handling scheme for both interactive virtual objects and system operation of VR/MR platform is needed. In this paper, we describe the three components, which are the direct manipulation interfaces for potable MR space and for fixed shared VR/MR space, and Interactive 3D Marker for displaying and manipulating virtual objects reflected lighting and geometry conditions of the real world. These components can give users seamless manipulation workspace by the natural operations such as they behave in their daily life. We also introduce Interactive Interior Design System using these components. This system realizes to coordinate the suitable interiors in the real space, by the free manipulation of virtual interior objects reflected the geometry and the lighting condition of the real space.
\end{abstract}

\section{Introduction}

There are many researches about the shared MR space for collaborative works such as conferencing, object modeling, layout planning and so on. However, most of the foregoing researches only connect two or few fixed sites with special multiple displays to provide immersive workspaces. Few user interfaces in the foregoing researches also allow users to manipulate MR space by the natural operations such as they behave in their daily life.

Our research projects aim to connect various VR/MR platforms as shown in Fig. 1 and to realize the seamless collaborative works with the intuitive operations whenever and wherever users like. In order to realize these spaces, it is need to develop two technologies: one is the portable MR workspace which users can carry out whenever they like, another is an effective handling scheme for both interactive virtual objects and operation of VR/MR platform. 
In this paper, we describe the three components, which are the direct manipulation interfaces for potable MR space and for fixed shared VR/MR space, and Interactive 3D Marker for displaying and manipulating virtual objects reflected lighting and geometry conditions of the real world. These components can give users seamless manipulation environment. We also introduce Interactive Interior Design System using these components. This system realizes to coordinate the suitable interiors in the real space, by the free manipulation of virtual interior objects reflected the geometry and the lighting condition of the real space.

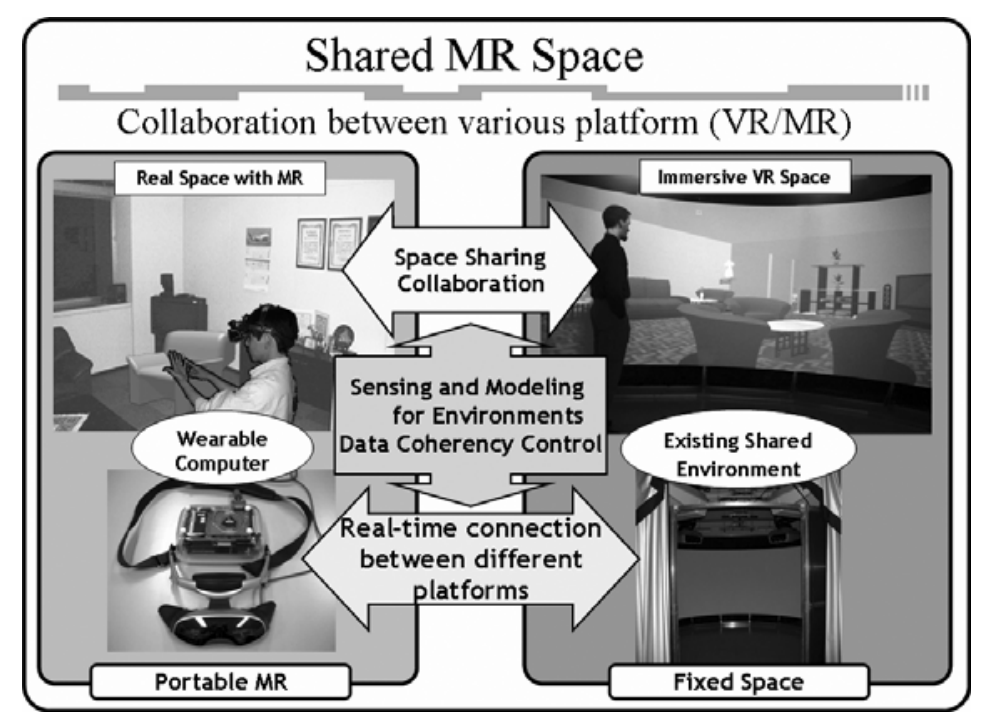

Fig. 1. Collaborative Works Between Various VR/MR Platforms

\section{Direct Manipulative User Interface}

\subsection{Hand Menu for Hands-Free Operation in the Portable MR Space}

In order to realize the portable MR spaces which gives users the MR workspace whenever and wherever they like, it is important to realize the easy and intuitive interface for MR space.

Hand Interface as shown in Fig. 2 is a non-contact and intuitive interface to realize an easy manipulation of a computer every time and everywhere. This interface only needs the user's hands and a camera attached with the head mounted display, without any additional device such as foregoing systems [1-4]. When the user performs some easy actions within the camera view, the user can operates the menu, selects some objects in the MR space, writes or draws, takes a picture, and so on.

Hand Menu is a menu system which is one module of Hand Interface. When the user opens his/her hand widely within the camera view, the system shows the user virtual menu items superimposed on his/her fingertips. The user can select a menu item 


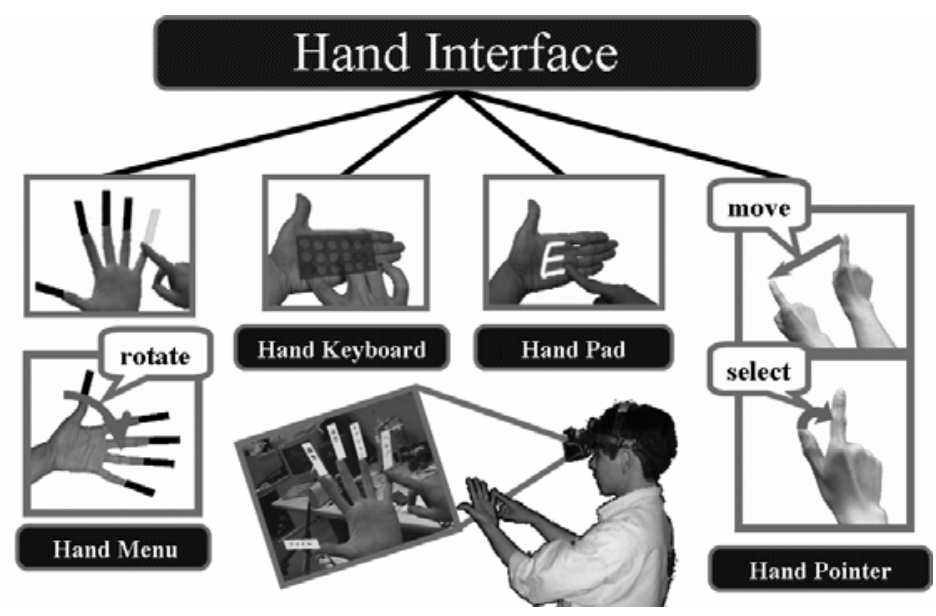

Fig. 2. Overview of Hand Interface

he/she wants to choose easily, by touching certain his/her fingertip superimposed a menu item with the index finger of another hand.

All of these processes are performed through the image recognition technique. Hand Menu recognizes the user operations by the following 6 steps:

1. hand-are extraction

2. fingertips detection

3. distinguishing the menu-hand superimposed menu items and the selection-hand to touch the menu

4. distinguishing the palm and the back of the menu-hand

5. displaying menu items

6. menu selection

At first, the system extracts hand-areas from obtained image through the camera using skin color information of the hand. In this hand-area, the system detects the fingertips utilizing a curvature through tracking the outline of the hand-area. The method to distinguish the menu-hand and the selection-hand is quite simple, using the number of detected fingertips. The system recognizes the region with five fingertips as the menu-hand and the region with one fingertip as the selection-hand. In order to distinguish the palm and the back, that is, whether the palm faces to the user's eye or not, the system examines the geometrical relation among the index, thumb and pinkie. As soon as the system recognizes that the menu-hand is exposed, the system superimposes the menu on each fingertip of the menu-hand in the user's sight through the seethrough HMD. According to the orientation of the menu-hand, one of two menu sets is displayed. The system recognizes that the user selects a certain menu item, using each position and relation of the fingertips of the menu-hand and the selection-hand, and starts up the selected menu. 


\subsection{Direct Manipulation Interface for Fixed Immersive VR/MR Space}

There are many researches how to manipulate the virtual objects and the VR/MR space [5-10]. However, it is difficult to allow users to manipulate them naturally just as if users manipulate the object of the real space in their daily life.

In order to realize the direct manipulation interface in the fixed immersive VR/MR environment, we are developing the free manipulation system using the infrared motion sensing system, as shown in Fig. 3.

This system allows users to move freely in the immersive environment and to manipulate the virtual objects naturally by the operations of touching, grasping, putting up, putting down, pointing and so on such as users behave in their daily life. The user has only to attach the Infrared Rays (IR) reflecting marker on the fixed points of his/her body and to enter this system.

When the user makes some action, the system obtains the 3D position of each IR maker from the obtained images through many IR cameras. Based on the 3D positions of the markers, the system assumes the 3D position of the user's body parts and recognizes how action he/she makes.

Here, we classify the user's action into 3 types:

1. whole action

2. local near action

3. local far action

Whole action is the action moving whole body such as going around in the immersive space, looking out and so on. In order to detect whole action, the user's position and direction in the immersive space is needed. So, we attach two IR markers to 3D eyeglass as shown in Fig. 4, and the system detects a middle point of the markers as the position of the user's head.

Local near action is the action when interacting virtual objects near the user, such as touching, grasping, putting up, putting down, pointing and so on. Local far action has similar aim as local near action. This is the action to manipulate virtual objects far from the user by combining pointing action and local near action. So, we attach IR markers to the hand for detecting local near action, elbow and shoulder for the pointing action as shown in Fig. 4.

The system detects the detail hand's action from the marker with the hand, and the pointing point by assuming from the point of hand, elbow, shoulder and head. In order to estimate the pointing point, the system uses the reference point $p, q$ and $r$, as shown in Fig. 5. Reference point $p, q$ and $r$ are the point on the line extended from head, elbow and wrist to the index fingertip. The system estimates the pointing point according to Eq. 1.

$$
a \vec{p}+b \vec{q}+(1-a-b) \vec{r}=\vec{O}
$$

Here, the vector $O$ is the position vector of target. The $a$ and $b$ are weight parameters to define the pointing point, which are obtained in advance using known points.

When the user indicates some point, the system calculates the pointing point recursively as the following. First, the system defines the reference point $p$ as initial value of interim point and obtains the weight in this point. The new interim point is calcu- 
lated by the reference point $p, q$ and $r$, and the weight. This calculation is performed until the difference between the previous interim point and the new interim point becomes below a threshold value.

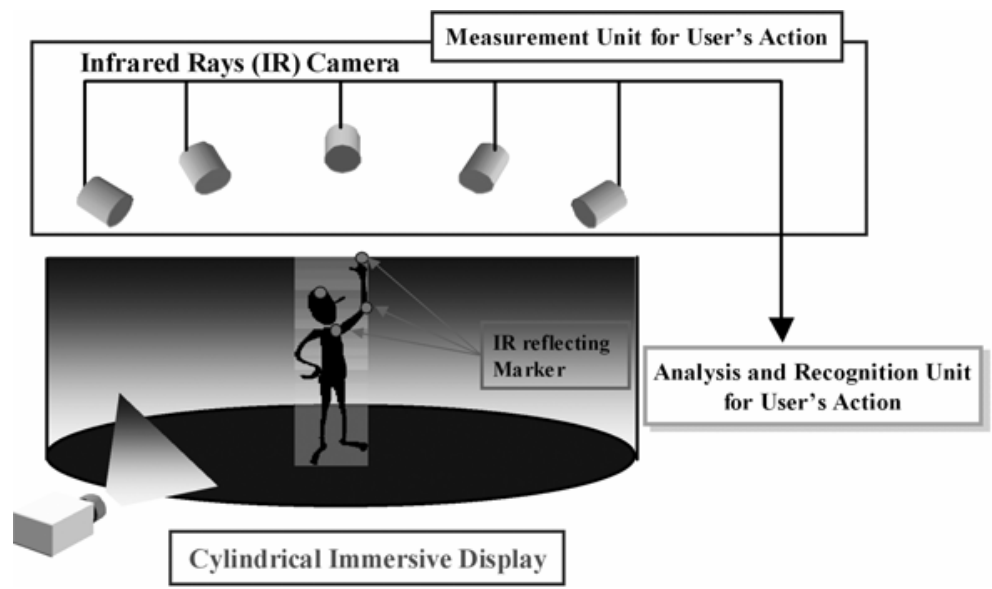

Fig. 3. System Overview

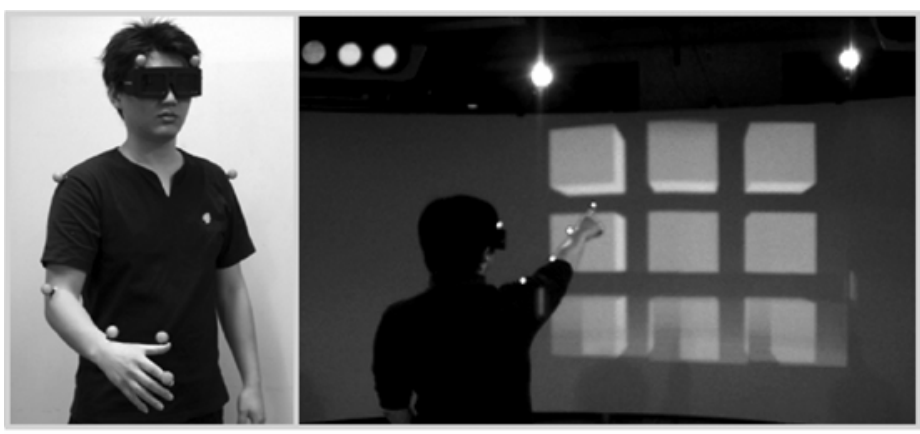

Fig. 4. Manipulation of the virtual objects by the natural motion

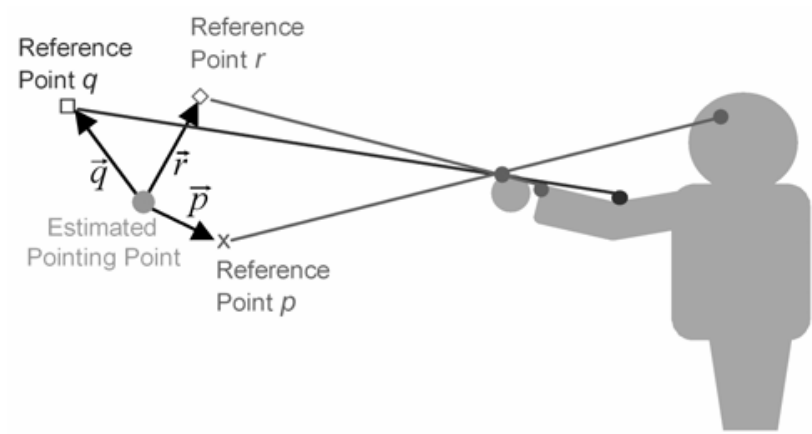

Fig. 5. Estimation of the Pointing Point 


\section{Interactive 3D Marker}

Interactive 3D Marker realizes to acquire geometric and photometric information simultaneously with low processing cost. This marker consists of a cube with four 2D codes on the side and a spherical mirror on the top, as shown in Fig. 6.

Interactive 3D Marker can obtain its position and posture from $2 \mathrm{D}$ codes and lighting condition from a spherical mirror. So, when a user manipulates this marker directly such as moves or rotates it, a virtual object assigned to this marker can be displayed immediately according to the lighting and geometry conditions in the real space.

We assign a unique pattern to each $2 \mathrm{D}$ code to use it to detect which side is seen from the camera. In this paper, we used $4 \times 4=16$ bits pattern, so that $\left(2^{16}-2^{8}\right) /(4 \times$ $4)=4080$ kinds of virtual objects can be assigned for 16 bits $2 \mathrm{D}$ code, considering excluding rotationally symmetric patterns.

Fig. 7 shows the process flow.

When a 2D code of Interactive 3D Marker is detected in the obtained image from the camera, the system calculates the geometrical information including its distance and posture from the appearance of the contour of the code region [11][12]. At the same time, the system acquires the embedded ID number by decoding the $2 \mathrm{D}$ code. Each ID number assigned to 2D code is associated with CG model. The CG models were defined as polygon models which contain thousands of vertices on which diffuse and specula reflection parameters were properly designed, in advance.

Continuously, the system calculates the lighting information from the spherical mirror of Interactive 3D Marker. The system assumes that lighting condition at the position of the 3D marker contains direct light from the light source, reflected light from specula surface in the environment and ambient light from distant view [13][14]. As shown in Fig. 8, when a spherical mirror is observed as a circle whose radius is $r$ pixels and center position is $\left(x_{c}, y_{c}\right)$, normal vector $N$ at the position $(x, y)$ on the sphere surface observed in the spherical mirror image is expressed as Eq. 2

$$
\begin{aligned}
& \vec{N}=\left[e_{x}, e_{y}, e_{z}\right] \\
& e_{x}=\frac{\left(x-x_{c}\right)}{r}, \quad e_{y}=\frac{\left(y-y_{c}\right)}{r}, \quad e_{z}=\sqrt{1-\left(e_{x}^{2}+e_{y}^{2}\right)}
\end{aligned}
$$

Light coming through the focal point $O$ into a pixel position $(x, y)$ is emitted from a direction which is regular reflection line relative to normal vector $N$ on the sphere. In the same way, the direction of the light source to illuminate each pixel on the image plane can be calculated.

$$
\vec{L}=\vec{V}-2(\vec{N} \bullet \vec{V}) \vec{N}
$$

The image of the spherical mirror is divided into small regions and sampled the intensity of the light source which illuminates each region. The light intensity is calculated by averaging $\mathrm{R}, \mathrm{G}$ and $\mathrm{B}$ value. The calculation of the direction of the light sources is used the centers of gravity of the regions. 
Finally, based on the obtained the geometry and lighting information, the system renders the CG models as fitting in the real space. The system assumes that the colors of the object surface are determined according to the model of Lambert and Phong for rendering virtual objects.
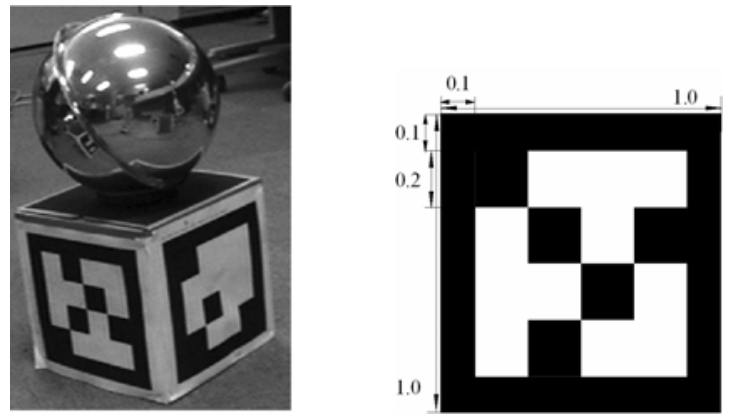

Fig. 6. Interactive 3D Marker (left) and 2D Code (right): digits stand for the size ratio

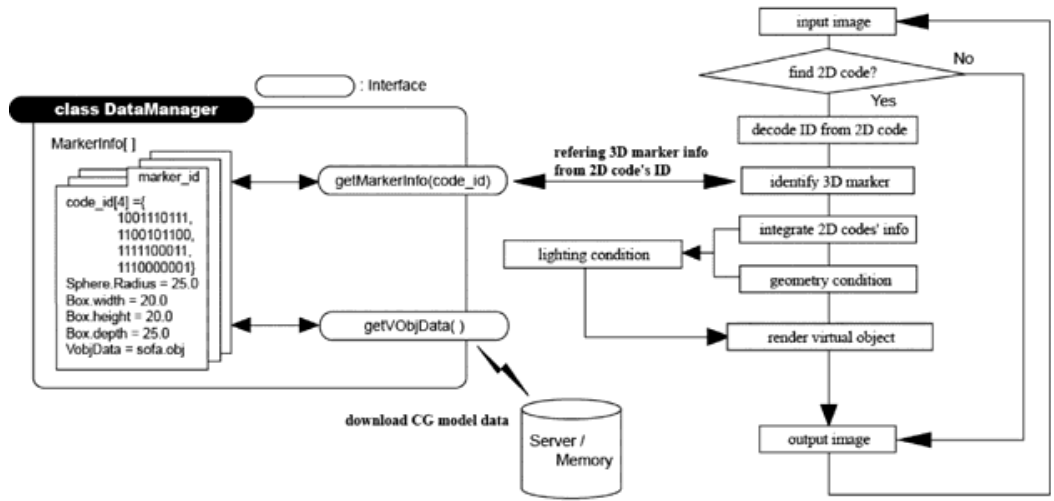

Fig. 7. Process Flow

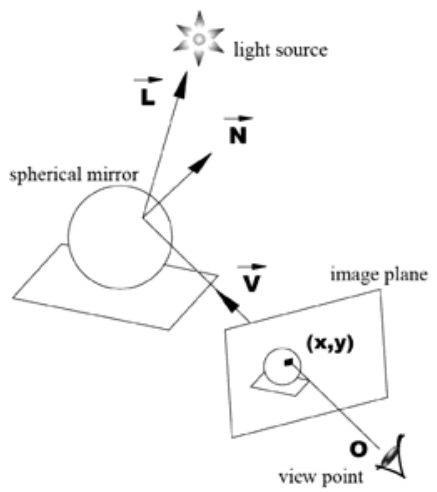

Fig. 8. Schematic Geometry of Viewpoint and Light Source 


\section{Interactive Interior Design System}

In this section, we describe the interior design system which is an application using the direct manipulative user interface and Interactive 3D Marker.

Fig. 9 shows the over view of the Interactive Interior Design System.

To use this system, the users have only to wear the head mounted display (HMD) attached a camera and a wearable computer as a processing unit. The users can make an interior design by putting and moving Interactive 3D Marker on the place they like freely. As each Interactive 3D Marker is assigned some interior object in advance, Interactive 3D Marker within the user's view through the HMD is displayed as the interior object which is a virtual object overlaid on Interactive 3D Marker according to the lighting and geometry condition of this marker in the real space.

The users can not only move Interactive 3D Marker but also reassign Interactive 3D Marker from a certain interior object to other interior object. When the user opens his/her hand widely within the user's view, the system calls up Hand Menu, as shown in Fig. 10. Hand Menu has the menu items as the interior object's name or icons. As shown in Fig. 10, when the user selects a certain menu item by touching a menu item with his/her index finger, the system reassigns a selected object to Interactive 3D Marker on which his/her eyes were focused and redraw a new interior object immediately.

Therefore, this Interactive Interior Design System enables the user to try putting on and off the interiors reflected the lighting and geometry condition of the real world and to coordinate the suitable interiors in his/her space.

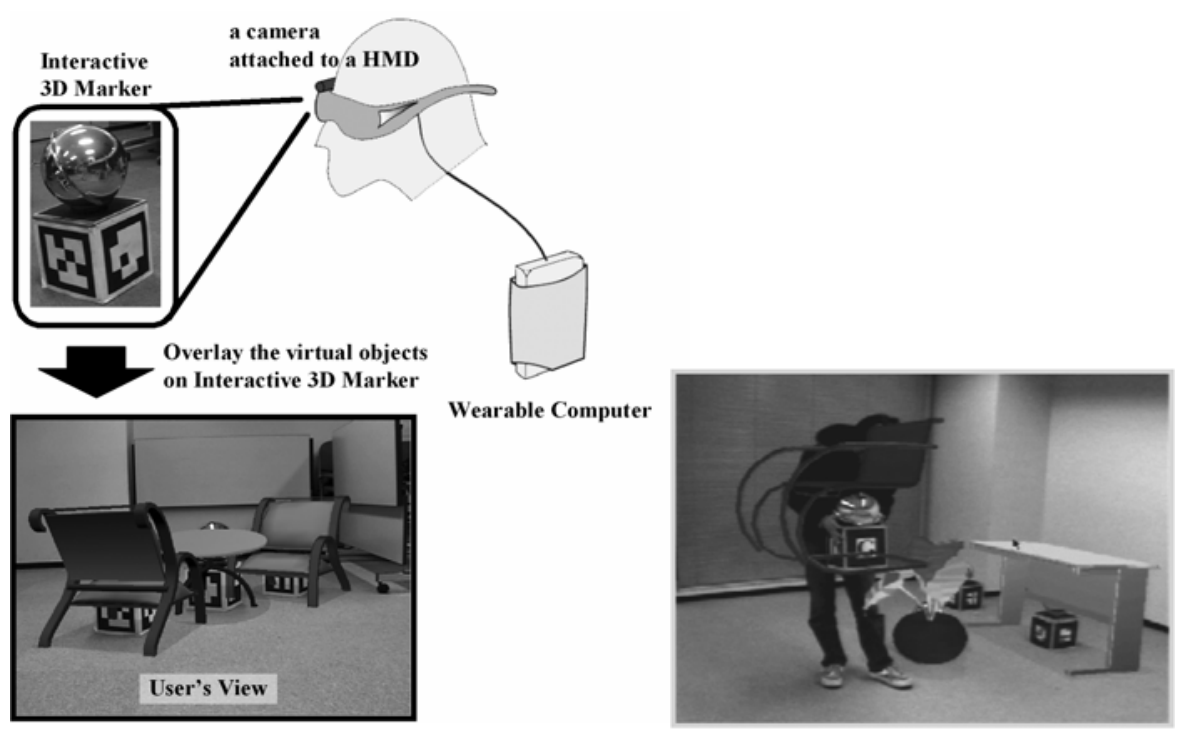

Fig. 9. Overview of Interactive Interior Design System 


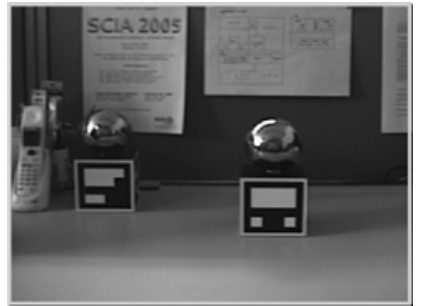

(a) Captured Image from Camera

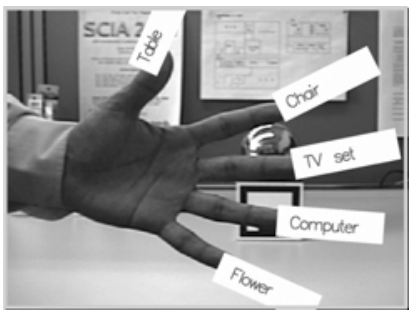

(c) Calling up Hand Menu

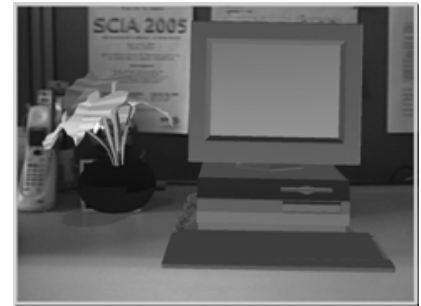

(b) Overlay the Virtual Objects (User's View)

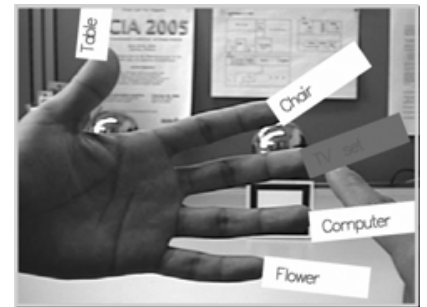

(d) Selecting a Menu Item

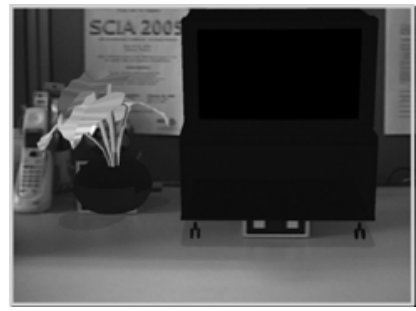

(e) Changed the Object to the Selected Item

Fig. 10. Menu Operation with Hand Menu

\section{Conclusions}

In this paper, we described Direct Manipulation Interface and Interactive 3D Markers, as an effective handling scheme in MR space. Hand Menu, direct manipulation interface for the portable MR space, realized the hands-free operation by the intuitive actions. For the fixed immersive VR/MR environment, we developed the natural manipulative interface such as user manipulates in the real space. Interactive 3D Marker realized to obtain the geometric and photometric information of the place put it on and to manipulate virtual objects freely and directly.

Furthermore, we described Interactive Interior Design System which was an application using Hand Menu and Interactive 3D Marker. This system realized to coordinate the suitable interiors in the real space, by the free manipulation of virtual interior objects reflected the geometry and the lighting condition of the real space and the hands-free menu operations. This system allowed several users to collaborate in the interior design wherever they wanted to make the interior design. This system gave the user seamless operations in the MR Space. 


\section{Acknowledgments}

This research is partly supported by Core Research for Evolutional Science and Technology (CREST) Program “Advanced Media Technology for Everyday Living” of Japan Science and Technology Agency (JST).

\section{References}

1. Masaaki Fukumoto, Yoshinobu Tonomura, Body Coupled FingeRing: Wireless Wearable Keyboard, Proceeding of the ACM Conference on Human Factors in Computing Systems, CHI97, pp. 147-154,1997.

2. Jun Rekimoto, GestureWrist and Gesture Pad: UnobtrusiveWearable Interaction Devices, Proceeding of the 5th International Symposium on Wearable Computers, pp. 21-27, 2001.

3. Koji Tsukada and Michiaki Yasumura: Ubi-Finger: Gesture Input Device for Mobile Use, Proceedings of APCHI 2002, Vol.1, pp.388-400, 2002.

4. Virtual Technologies, CyberGlove.

5. P.Hanrahan, L.D.Culter, B.Frolich: Two-handed Direct Manipulation on the Responsive Workbench, Symposium on Interactive 3D Graphics, pp.107-114, 1997.

6. T.Ilmonen: Immersive 3D User Interface for Computer Animation Control, International Conference of Computer Vision and Graphics, 2002.

7. I.Cohen and M.W.Lee: 3D Body Reconstruction for Immersive Interaction, 2nd International Workshop on Articulated Motion and Deformable Objects, pp.119-130, 2002.

8. S.Rougeaux, R.G.O'Hagan, A.Zelinsky: Visual Gesture Interface for Virtual Environments, User Interface Conference, pp.73-80, 2002.

9. D.Minnen, T.Westyn, A.Hurst, T.Starner, B.Leibe and J.Weeks: The Perceptive Workbench: Computer-vision-based Gesture Tracking, Object Tracking, and 3D Reconstruction for Augmented Desks, Machine Vision and Application, Vol.14, pp.59-71, 2003.

10. G.Wesche: The toolfinger: Supporting Complex Direct Manipulation in Virtual Environments, Proceedings of the workshop on Virtual Environments 2003 (EGVE '03), pp.39-45, 2003.

11. H.Kato and M.Billinghurst, Marker tracking and HMD calibration for a video based augmented reality conferencing system, IEEE International Workshop on Augmented Reality, pp. 125-133, 1999.

12. Diego Lopez de Ipi na, Paulo Mendonca, and Andy Hopper : "a low-cost visionbased location system for ubiquitous computing", Personal and Ubiquitous Computing, Vol. 6, No. 3, pp. 206-219, 2002.

13. Paul Debevec, Rendering synthetic objects into real scenes: Bridging traditional and image-based graphics with global illumination and high dynamic range photography, Proceedings of SIGGRAPH 98, pp. 189-198, 1998.

14. I.Sato, Y.Sato, and I.Ikeuchi, Acquiring a radiance distribution to superimpose virtual objects onto a real scene, IEEE Transactions on Visualization and Computer Graphics, Vol. 5, No. 1, pp. 1-12, 1999. 